\title{
NEDD8 conjugation in Schistosoma mansoni: Genome analysis and expression profiles
}

\author{
Roberta V. Pereira ${ }^{\text {a }}$, Matheus de S. Gomes ${ }^{\text {b }}$, Roenick P. Olmo a , Daniel M. Souza ${ }^{\text {, }}$ Liana K. Jannotti-Passos ${ }^{\text { }}$, \\ Elio H. Baba ${ }^{\mathrm{c}}$, William Castro-Borges ${ }^{\mathrm{a}}$, Renata Guerra-Sá ${ }^{\mathrm{a}, *}$ \\ a Núcleo de Pesquisas em Ciências Biológicas, Universidade Federal de Ouro Preto, Morro do Cruzeiro, Ouro Preto, MG, Brazil \\ b Instituto de Genética e Bioquímica, Universidade Federal de Uberlândia Campus Avançado Patos de Minas, MG, Brazil \\ c Centro de Pesquisas René Rachou, Fiocruz, Belo Horizonte, MG, Brazil
}

\section{A R T I C L E I N F O}

\section{Article history:}

Received 27 June 2012

Received in revised form 18 December 2012

Accepted 22 December 2012

Available online 8 January 2013

\section{Keywords:}

NEDD8

NEDDylation

Schistosomula development

Differential expression

\begin{abstract}
A B S T R A C T
NEDD8 is an ubiquitin-like molecule that covalently binds to target proteins through an enzymatic cascade analogous to ubiquitylation. This modifier is known to bind to p53 and p73, as well as all Cullin family proteins, which are essential components of Skp1/Cul-1/F-box protein (SCF)-like Ub ligase complexes. Here, we focused on a genomic analysis of the genes involved in the NEDD8 conjugation pathway in Schistosoma mansoni. The results revealed seven genes related to NEDD8 conjugation that are conserved in Schistosoma japonicum, Caenorhabditis elegans, Drosophila melanogaster and Homo sapiens. We performed quantitative RT-PCR (qRT-PCR), which showed differential profiles for Smnedd8, Smapp1, Smuba3, Smube2f, Smdcn1, Smrbx and Smsenp8 throughout the life cycle of S. mansoni. Upregulation was observed in 3-day-old schistosomula and adult worms for all analysed genes. We also analysed the transcription levels of Cullin family members Smp63 and Smp73, and observed upregulation in early schistosomula, while cercariae and adult worms showed expression levels similar to one another. Taken together, these results suggest that the NEDDylation/ DeNEDDylation pathway controls important cellular regulators during worm development from cercariae to schistosomula and, finally, to adult.
\end{abstract}

(c) 2013 Elsevier Ireland Ltd. All rights reserved.

\section{Introduction}

Posttranslational modifications (PTMs), such as ubiquitylation and ubiquitin-like protein (Ubl) modifications, play critical roles in a variety of cellular processes. The ubiquitin-proteasome pathway is one of the best-characterised systems involved in protein regulation in eukaryotes [1,2]. A number of small Ubls such as SUMO (Small ubiquitin modifier), NEDD8 (neural precursor cell-expressed developmentally downregulated-8) and ISG15 (interferon-induced ubiquitinlike modifier) have been found covalently attached to their targets in a manner similar to the ubiquitylation process. Among the Ubls, the NEDD8 protein has been found to be evolutionarily conserved in eukaryotes and has the highest identity with the ubiquitin protein (59\%) $[3,4]$. Studies have revealed that NEDD8 is essential for cell viability in fission yeast and is required for development processes in both mouse and Drosophila [5-7].

The NEDDylation pathway, analogous to the ubiquitin pathway, involves a set of enzymes working together to conjugate the NEDD8 protein to specific target proteins [8-10]. In the first step, the NEDD8-

\footnotetext{
* Corresponding author at: Universidade Federal de Ouro Preto, Departamento de Ciências Biológicas/Núcleo de Pesquisas em Ciências Biológicas, Instituto de Ciências Exatas e Biológicas, ICEB2, Sala 045, Campus Morro do Cruzeiro, 35400-000, Ouro Preto, MG, Brazil. Tel.: +55 31 35591697; fax: +55 3135591680 .

E-mail address: rguerra@iceb.ufop.br (R. Guerra-Sá).
}

specific E1 activating enzyme (APP-BP1/Uba3 heterodimer) activates the NEDD8 protein by adenylating the carboxyl terminal glycine residue. This is followed by the formation of a high-energy thioester bond with the catalytic cysteine residue of UBA3. In the next step, NEDD8 is transferred from the E1 enzyme to a catalytic cysteine residue of the NEDD8-specific E2 conjugation enzymes UBC12 (also known as UBE2M) or UBE2F, again using a thioester linkage. Finally, the activated NEDD8 on the E2 enzyme is subsequently transferred and conjugated to the E3 ligase substrates. The RING-finger protein ROC1, a subunit of the Cullin Ring Ligases (CRLs), interacts with UBC12, acting as the NEDD8 E3 ligase for Cullins. The most significant and best-studied substrates of NEDD8 are the Cullin proteins, which serve as scaffolds in E3 CRLs [11,12].

NEDD8 contains seven conserved lysine residues, similar to the ubiquitin protein. The NEDD8 residues K11, K22, K48 and K60 can allow NEDD8 to form chains in vivo, whereas the NEDD8 residues K22 and K48 can be NEDDylated in vitro, suggesting that chain formation of ubiquitin and ubiquitin-like proteins might be a general phenomenon for these modifications [13]. In addition, the NEDD8 modification is a dynamic and reversible process that is controlled by NEDD8-specific proteases that remove NEDD8 protein from distinct targets. A specific activity of the NEDD8-specific protease has been reported to be associated with a metalloprotease motif in JAB1/CSN5, a subunit of the COP9 signalosome. The whole COP9 signalosome complex is required for CSN5 activity, as evidenced by the fact that the isolated protein did not display NEDD8 protease 
activity $[14,15]$. NEDD8, similar to the UBL proteins, is synthesised as an inactive precursor and has to be processed by a NEDD8-specific protease (SENP8). This processing exposes the diglycine motif at the carboxyl-terminus, which is required for the conjugation process. SENP8 is a highly conserved and specific protease for NEDD8. Neither ubiquitin nor SUMO are utilised as SENP8 substrates, although both share $\mathrm{COOH}$-terminal extensions [16].

Although the NEDDylation process shares similarities to the ubiquitylation process, the biological impact of this modification appears to be different. Ubiquitylation has been shown to be involved in trafficking, signalling, endocytosis and proteasome-dependent proteolysis, whereas NEDDylation has been implicated in modulation of the biochemical activity of target proteins to affect their stabilities [17]. Recently, a set of NEDD8 target proteins have been identified, including pVHL, p53, BCA3, the EGF receptor and ribosomal proteins [18].

Previous investigations revealed the importance of ubiquitylation during S. mansoni development $[19,20]$ and evidenced the complexity of the proteasome-dependent intracellular protein degradation in adult worms [21]. Subsequently, our group reported the existence of two SUMO paralogs and UBC9 in S. mansoni, confirming the importance of these posttranslational protein modifications in this parasite $[22,23]$. In this work we aimed to investigate the importance of posttranslational modifications in this parasite, focusing on the NEDDylation system. We analysed the conservation of the proteins predicted to be related to this pathway using $S$. mansoni databases. We then evaluated the gene expression of the components of the NEDD8 machinery, as well as the putative NEDD8 targets (Cullins, p63 and p73), using qRT-PCR at the cercariae, adult worm and mechanically transformed schistosomula (MTS) stages of development. The current study provides for the first time information about the NEDDylation machinery in the S. mansoni parasite. Investigations involving posttranslational regulation may help to better understand the biology of the parasite, as well as its relationship with the host.

\section{Materials and methods}

\subsection{Ethics statement}

All experiments involving animals were authorised by the Ethical Committee for Animal Care of Federal University of Ouro Preto and were in accordance with the national and international regulations accepted for laboratory animal use and care.

\subsection{Parasites}

The LE strain was maintained by routine passage through Biomphalaria glabrata snails and BALB/c mice. The infected snails were induced to shed cercariae under light exposure for $2 \mathrm{~h}$ and the cercariae were recovered by sedimentation on ice. Adult worm parasites were obtained by liver perfusion of mice after 50 days of infection. Mechanically transformed schistosomula (MTS) were prepared as described by Harrop and Wilson [24]. Briefly, cercariae were recovered and washed in RPMI 1640 medium (Invitrogen) before vortexing at maximum speed for $90 \mathrm{~s}$ and then were immediately cultured for $3.5 \mathrm{~h}$ at $37^{\circ} \mathrm{C}$, $5 \% \mathrm{CO}_{2}$. The recovered schistosomula were then washed with RPMI 1640 until no tails were detected. For subsequent incubations, the parasites were maintained in M169 medium supplemented with 10\% FBS, $100 \mu \mathrm{g} / \mathrm{mL}$ penicillin, $100 \mu \mathrm{g} / \mathrm{mL}$ streptomycin and 5\% Schneider's medium [25] at $37{ }^{\circ} \mathrm{C}$ on $5 \% \mathrm{CO}_{2}$ for $3.5 \mathrm{~h}, 1,2,3,5$ and 7 days.

\subsection{Identification and computational analysis of the NEDDylation pathway and possible targets}

The sequences for SmNEDD8, SmAPP1-UBA3, SmUBE2F, SmDCN1, SmRBX, SmSENP8 and possible targets (SmCullins, Smp63 and Smp73) were retrieved through BLAST searches from the $S$. mansoni genome database version 5.0, available at http://www.genedb.org/genedb/ smansoni/. Amino acid sequences from the orthologs in Drosophila melanogaster, Caenorhabditis elegans and Homo sapiens were used as queries. The BLASTp algorithm, underpinned by Pfam (v26.0), allowed detection of conserved protein domains or motifs from $S$. mansoni sequences. Multiple alignments of SmUBE2F and SmCullins were performed by ClustalX 2.0 [26]. Phylogenetic analysis was conducted by MEGA 5 [27]. A phylogenetic tree of these sequences was inferred using the Neighbor-Joining method [28]. The bootstrap consensus tree inferred from 1000 replicates was used to represent the evolutionary history of the taxa analysed. Branches corresponding to partitions reproduced in less than $50 \%$ of the bootstrap replicates are collapsed. The percentage of replicate trees in which the associated taxa clustered together in the bootstrap test (1000 replicates) is shown next to the branches. The tree was drawn to scale, with branch lengths in the same units as those of the evolutionary distances used to infer the phylogenetic tree. All positions containing gaps and missing data were eliminated from the dataset.

\subsection{Expression analysis of NEDDylation pathway components and possible targets}

Total RNA from adult worms, cercariae and schistosomula were obtained using a combination of the Trizol reagent (GIBCO-BRL) and chloroform for extraction and then were on-column purified using the "SV total RNA Isolation System" (Promega, Belo Horizonte, Brazil). The preparation was treated with 3 different rounds of RNase-free DNase I (RQ1 DNase; Promega). RNA was quantified using a spectrophotometer and an aliquot containing $1 \mu \mathrm{g}$ of total RNA reverse transcribed using an oligodT primer from the Thermoscript RT-PCR System (Invitrogen São Paulo, Brazil), as described by the manufacturer. The efficiency of DNAse I treatment was evaluated by PCR amplification of the cDNA reaction mix without the addition of the Thermoscript enzyme. S. mansoni-specific primers were designed using the program GeneRunner ${ }^{\circledR}$. The sequence accession numbers and their pair of primers are in the supplementary materials (Table S1). Reverse-transcribed cDNA samples were used as templates for PCR amplification using SYBR Green Master Mix UDG-ROX® (Invitrogen) and the 7300 Real Time PCR System (Applied Biosystems, Rio de Janeiro, Brazil). Specific primers for S. mansoni EIF4E were used as an endogenous control (GeneDB ID: Smp_001500) (forward 5' TGTTCCAACCACGGTCTCG3', reverse 5'TCGCCTTCCAATGCTTAGG3') [29]. The efficiency of each pair of primers was evaluated according to the protocol developed by the Applied Biosystems application (cDNA dilutions were $1: 10,1: 100$ and 1:1000). For all investigated transcripts three biological replicates were performed. The gene expression was normalised to the SmEIF4E transcript using the Applied Biosystems 7300 software according to the $2^{-\Delta \mathrm{Ct}}$ method [30].

\subsection{Statistical analysis}

Statistical analysis was performed using the GraphPad Prism version 5.0 software package (Irvine, CA, USA). Normality of the data was established using one way analysis of variance (ANOVA). Tukey post tests were used to investigate significant differential expression of transcripts throughout the investigated stages. In all cases, the differences were considered significant when $p$ values were $<0.05$.

\section{Results}

\subsection{NEDDylation pathway conservation in S. mansoni}

Using both a homology-based method and de novo prediction on S. mansoni sequences deposited in the genome database, we were able to characterise the components of the NEDDylation machinery in silico. This analysis identified seven genes involved in the NEDDylation 
mechanism in this parasite. These genes were the following: SmNEDD8 (GeneDB ID: Smp_130170), components of the E1 activation heterodimer SmAPP1 (GeneDB ID: Smp_087920) and SmUBA3 (GeneDB ID: Smp_151760), E2 conjugating enzyme SmUBE2F (GeneDB ID: Smp_ 027370), E3 ligases SmDCN1 (GeneDB ID: Smp_105330) and SmRBX (GenBank ID: DQ466078.1) and protease SmSENP8 (GeneDB ID: Smp_121890). The number of genes found in S. mansoni that were related to the NEDDylation pathway was compared with the number of genes from $S$. japonicum, $C$. elegans, D. melanogaster and $H$. sapiens. Our results demonstrate that the $S$. mansoni genes are very similar to those found in the Protostome organisms C. elegans and D. melanogaster, and identical to $S$. japonicum, reinforcing that the NEDDylation pathway is conserved in these organisms.

The conserved domains of SmNEDD8, SmUBE2F and SmSENP8 were assigned in these enzymes and compared with their orthologs in D. melanogaster, C. elegans and H. sapiens (Fig. 1). The predicted SmNEDD8 protein has 77 amino acid residues and contains a main domain, Rad60 (PF 11976). The predicted SmUBE2F protein has 186 amino acid residues and a UQ domain (PF 00179), characteristic of conjugation enzymes. Furthermore, SmUBE2F contains a critical

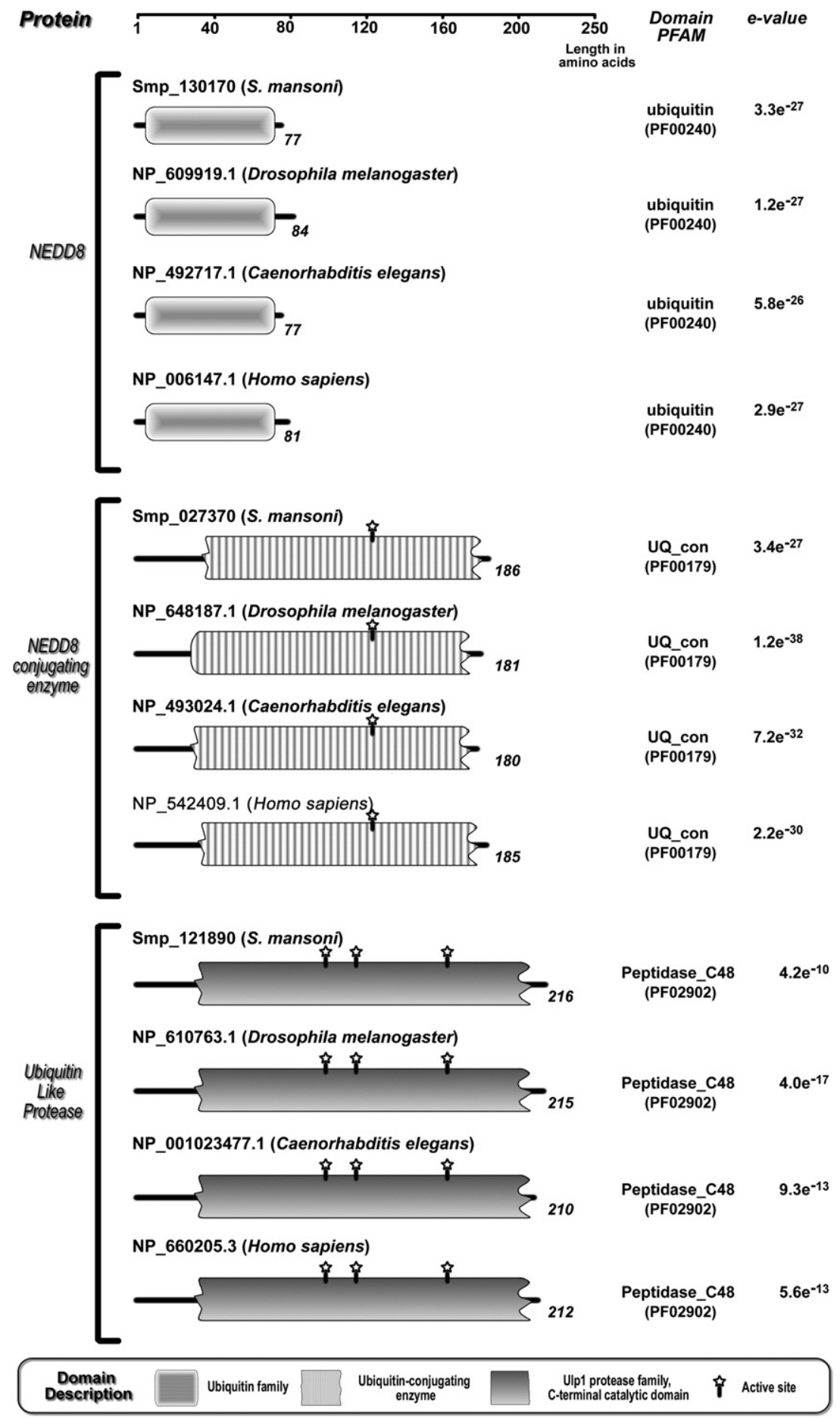

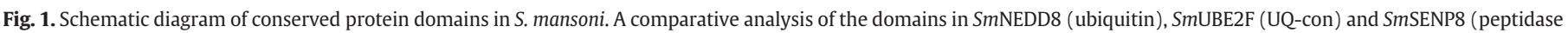
C-48) with their orthologs in D. melanogaster, C. elegans and H. sapiens. 


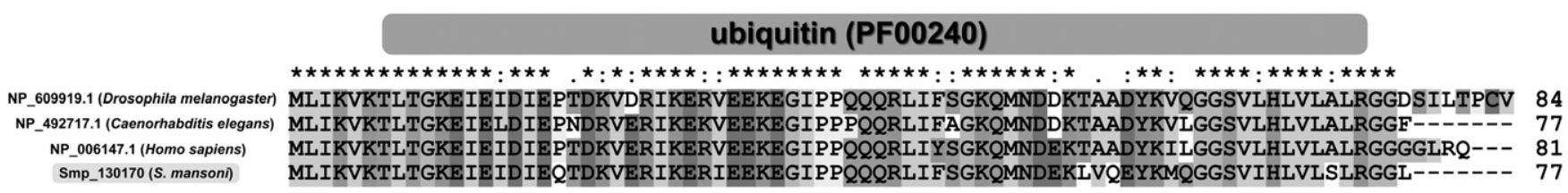

Fig. 2. Global alignment of SmNEDD8 and its orthologs, using the ClustalX 2.0 program and boxshade. Identical residues are shaded in black and similar residues in grey.

Cysteine residue at position117, similar to orthologous E2 conjugation enzymes. The predicted protein SmSENP8 has 216 amino acid residues and a conserved C-48 domain (PF 02902), which includes the enzyme active site residues histidine, aspartic acid and cysteine at the positions 102, 119 and 162, respectively (Fig. 1). Comparing SmNEDD8 with its orthologs, we noticed that this protein was highly conserved with at least $74 \%$ identity when compared to the proteins from D. melanogaster, C. elegans and H. sapiens (Fig. 2). The alignment of the proteins from S. mansoni and S. japonicum displayed $100 \%$ identity, affirming the evolutionary conservation of this species (data not shown). The gene encoding the NEDD8 E2 conjugation enzyme has been found duplicated in several organisms (UBE2F and UBE2M or UBC12 isoforms). The phylogenetic analysis displayed that the NEDD8$\mathrm{E} 2$ enzyme in S. mansoni is unique and has a higher homology with UBE2F orthologs than UBE2M orthologs (Fig. 3). The global alignment between E2-NEDD8 orthologs reinforced this conservation and the differences between the two E2 conjugation enzymes. The cysteine residue characteristic of the UQ_con domain is shown highlighted (Fig. 4).

\subsection{NEDDylation machinery is differentially regulated in S. mansoni}

The gene expression of the NEDDylation pathway components was analysed using the qRT-PCR technique with three biological replicates. The results were expressed using RNA levels in relation to EIF4E, a constitutively expressed gene. Regarding the expression of Smnedd8, we observed a 1.5-fold increase in MTS-3 d compared to the other developmental stages and similar levels when comparing cercariae to adult worm (Fig. 5A). The expression of Smapp1 and Smuba3 subunits that form the NEDD8-activating E1 enzyme demonstrated no relevant changes among the subunits. Both components of the E1 heterodimer were upregulated at least 2-fold in MTS-3.5h, 1d, 3 days and adult worm (Fig. 5B). The conjugation enzyme UBE2F is the only enzyme able to conjugate NEDD8 in S. mansoni. While verifying the expression of this transcript in the parasite stages, we found an increase of 2-fold in MTS-3.5h in relation to other schistosomula and an increase of 1.5 -fold in adult worm compared to cercariae (Fig. 5C). The expression of the E3 ligases (Smdcn1 and Smrbx) was very similar in all stages, except in adult worms, where RBX was upregulated 2-fold compared to other developmental stages (Fig. 5D). The NEDD8-specific protease SENP8 has been found to be capable of both processing the inactive precursor of NEDD8 and freeing NEDD8 monomers from the substrates they are bound to [18]. There was a stage-specific expression pattern of this enzyme's transcript during the cercariae-schistosomula transition and a 3-fold increase in MTS-1d and adult worm compared to other evolutionary stages (Fig. 5E). Together, the results showed a higher expression of all NEDDylation machinery components in adult worm, suggesting that this modification pathway is important during this stage of the parasite cycle.

\subsection{Cullins and tumour suppressor proteins (p63 and p73) are highly expressed in MTS-3.5h}

We identified five Cullins and their conserved domains in S. mansoni: SmCullin1 (GeneDB ID: Smp_150270), SmCullin2 (GeneDB ID:

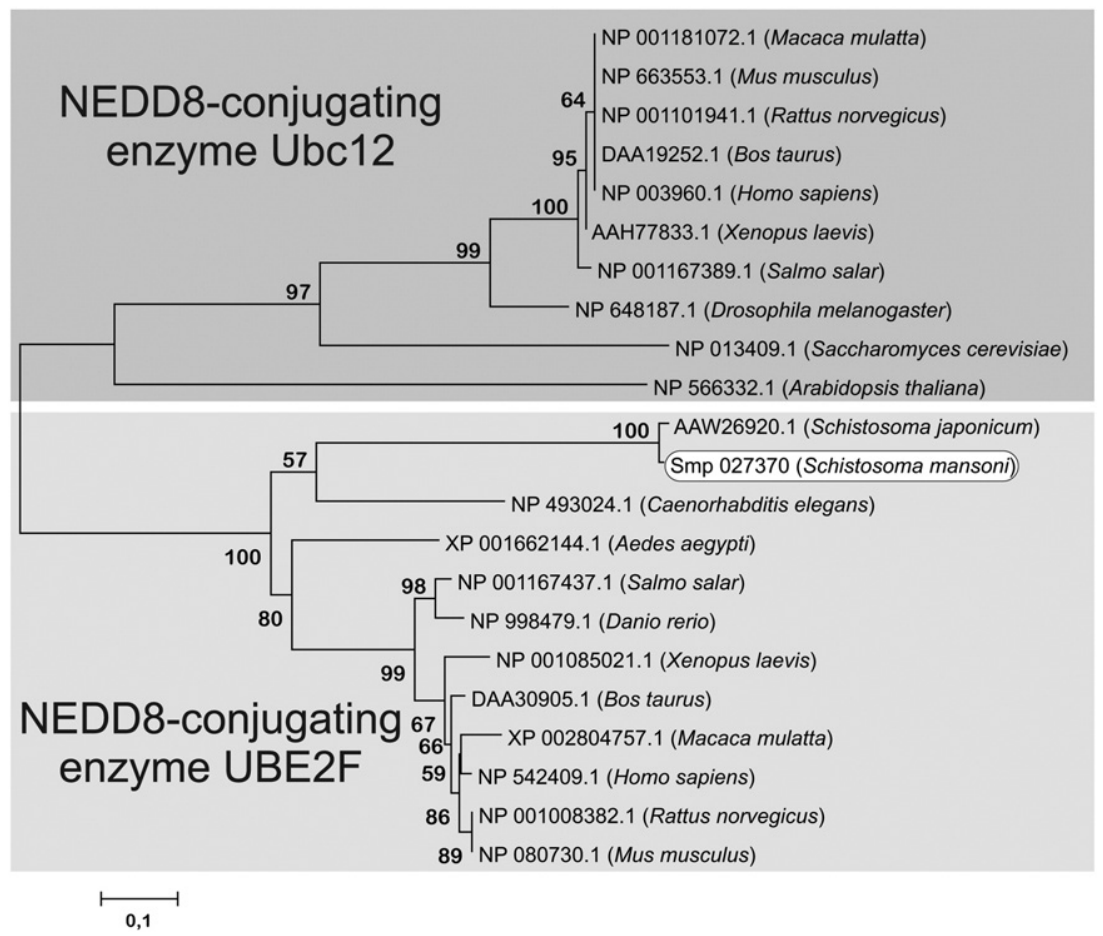

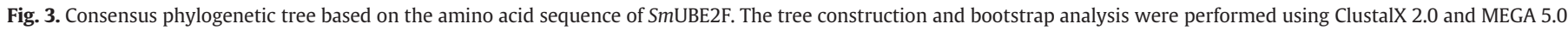

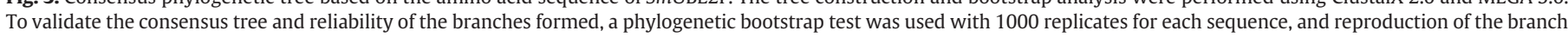
in $50 \%$ of the runs considered the minimum for a reliable branch. 
NP_542409.1 Homo_sapiens NP 001008382 . 1 . DAA30905.1_Bos_taurus

NP_-998479.1 Danio rerio NP_001167437.1_Salmo_salar XP_001662144.1_Aedes_aegypti AAW26920.1_Schistosoma japonicum NP_493024.1_Caenorhabditis_elegans NT_001181072.1_Macaca_mulatta NP_603553.1 Mus_musculus AAH77833.1_Xenopus_laevis NP_001167389.1_Salmo_salar NP 6013407.1 - Drosophila_melanogaste NP_566332.1_Arabidopsis_thalian

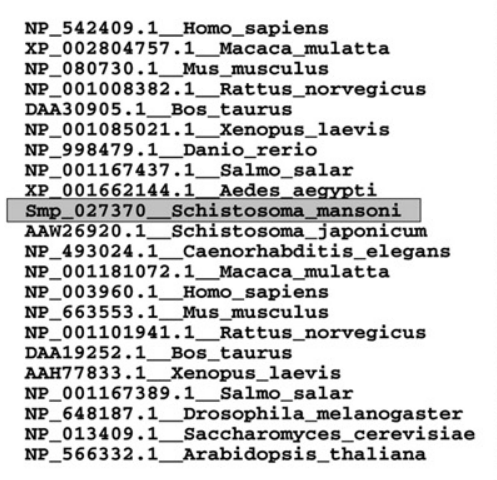

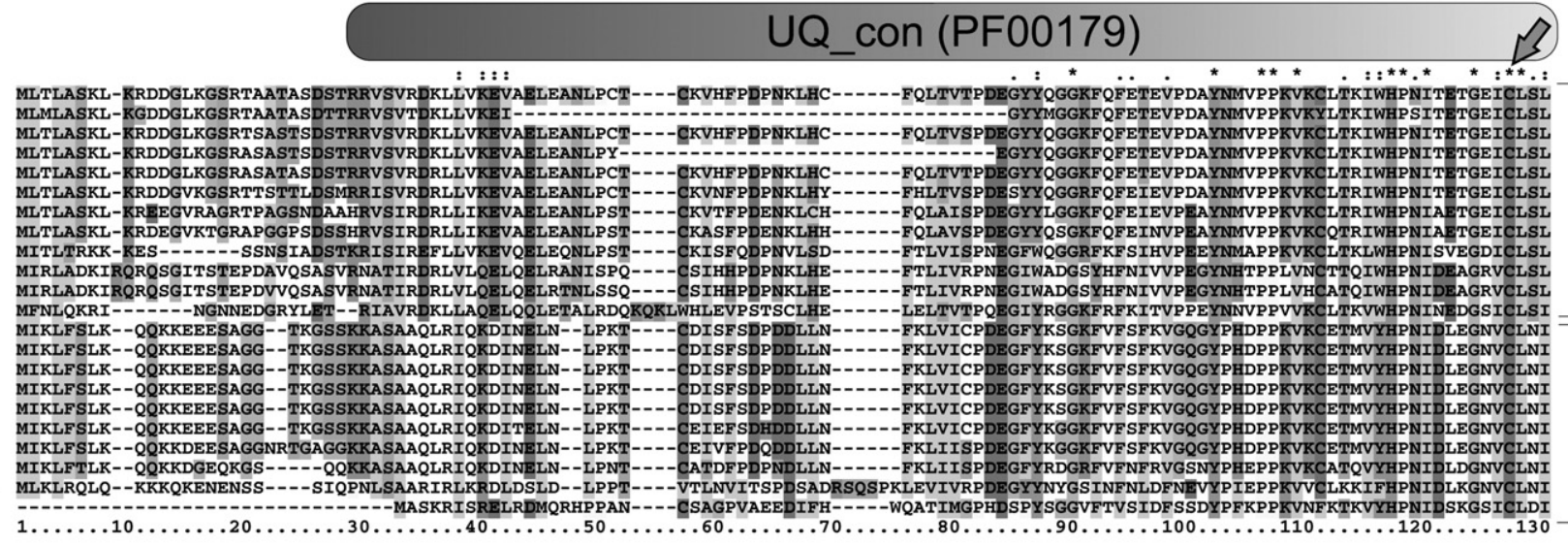

NEDD8-conjugating enzyme UBE2F

NEDD8-conjugating enzyme Ubc12

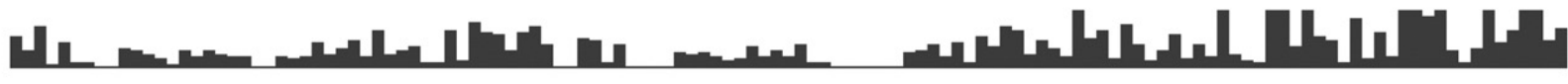

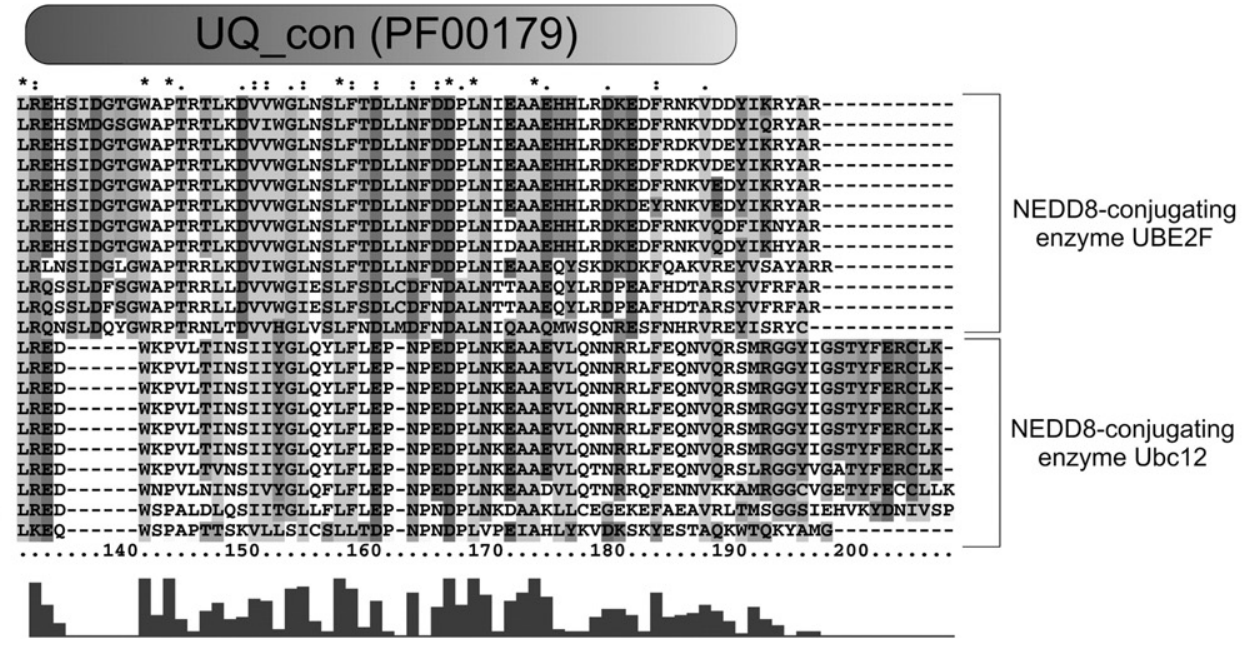

Fig. 4. CLUSTALX 2.0 multiple alignment of predicted amino acid sequences of SmUBE2F, showing the putative conserved domain UQ_con (PF00179), obtained by using the Pfam algorithm. 

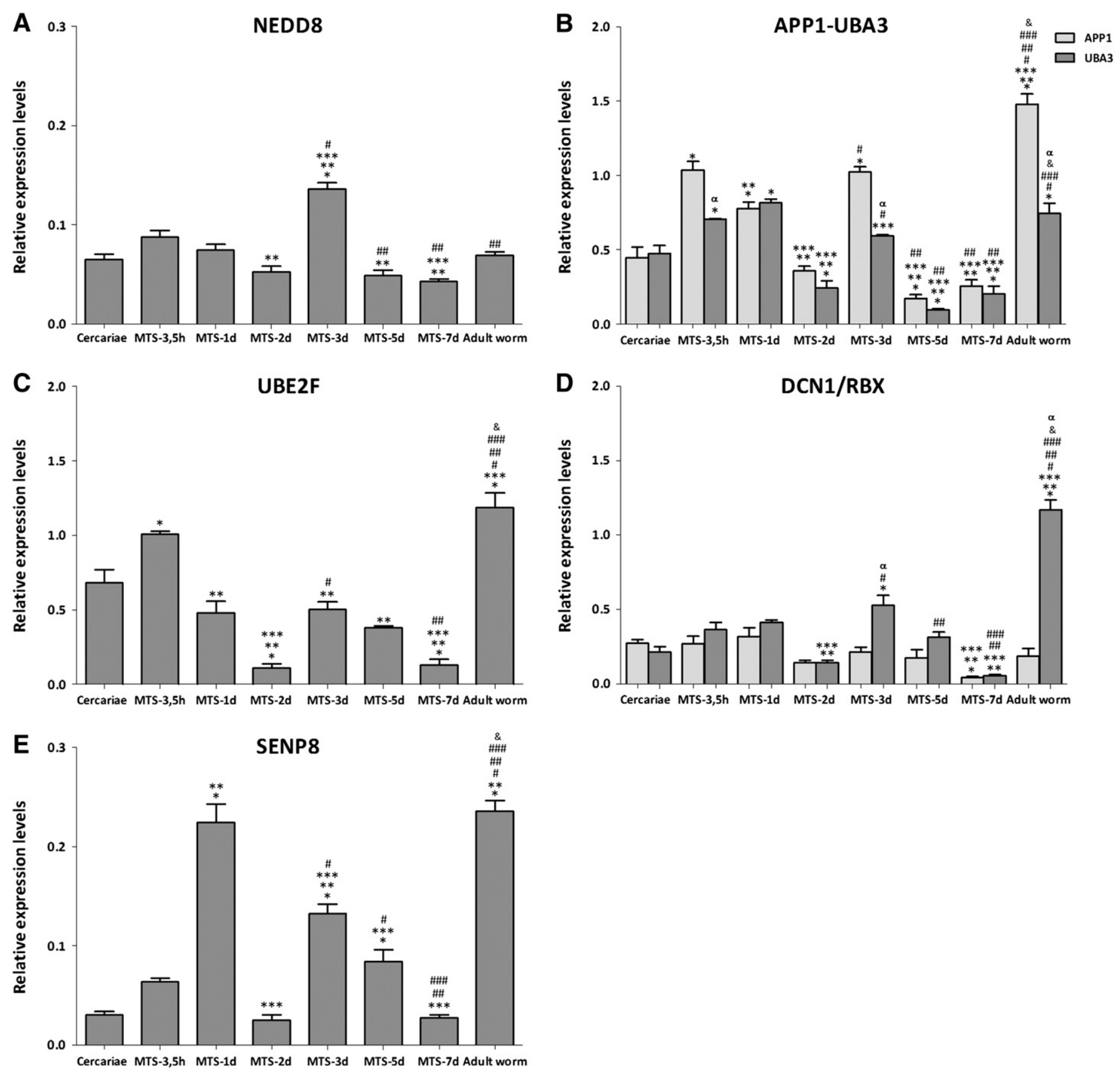

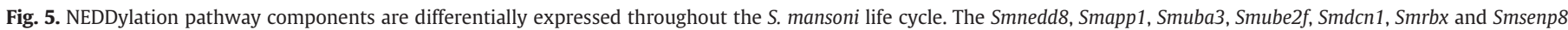

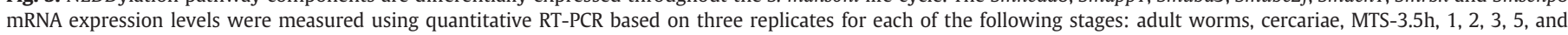

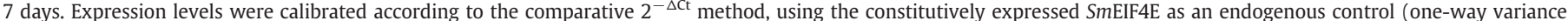

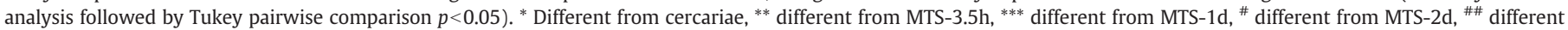
from MTS-3d, ${ }^{\# \# \# ~ d i f f e r e n t ~ f r o m ~ M T S-5 d ~ a n d ~}{ }^{\delta}$ different from MTS-7d. $t$ Tests were performed to compare UBA3 to APP1 and RBX to DCN1 ( ${ }^{\alpha}$ different from APP1 or DCN1).

Smp_079740), SmCullin3 (GeneDB ID: Smp_117580), SmCullin4 (GeneDB ID: Smp_082850) and SmCullin5 (GeneDB ID: Smp_143030). The phylogenetic analysis indicated that the Cullins' sequences are conserved among their orthologs (Fig. 6). We analysed the expression levels of these Cullins in cercariae, adult worms, MTS-3.5h, 1, 2, 3, 5 and 7 days. In MTS-3.5h, the levels of these Cullins were upregulated approximately 3-fold when compared with other stages. Smcullin 2, 3, 4 and 5 were expressed at the same levels in cercariae and adult worm stages (Fig. 7A-E). Regarding Smcullin1, we verified upregulation of approximately 2-fold in cercariae in relation to adult worms.

Furthermore, we analysed the two tumour suppressor proteins Smp63 (GeneDB ID: Smp_139530) and Smp73 (GeneDB ID: Smp_
136160.2), which have been found to be putative targets of the NEDD8 conjugation pathway (Fig. 7F). The p63 protein was characterised in S. mansoni and its expression was observed in adult worm [31]. In this article, this protein was denominated as p53. However, a recent study revealed that invertebrates do not have p53, only the orthologs p63 and p73 [32]. Therefore, in this article we used p63 instead of p53. The p63 and p73 proteins had similar expression levels to one another at all analysed stages. Both proteins were upregulated in MTS-3.5h and MTS-5 d (2-3-fold) in relation to the other analysed stages. Smp63 transcript was 3-fold higher in adult worm in relation to cercariae, whereas Smp73 mRNA was found upregulated 2-fold in cercariae compared to adult worm. 


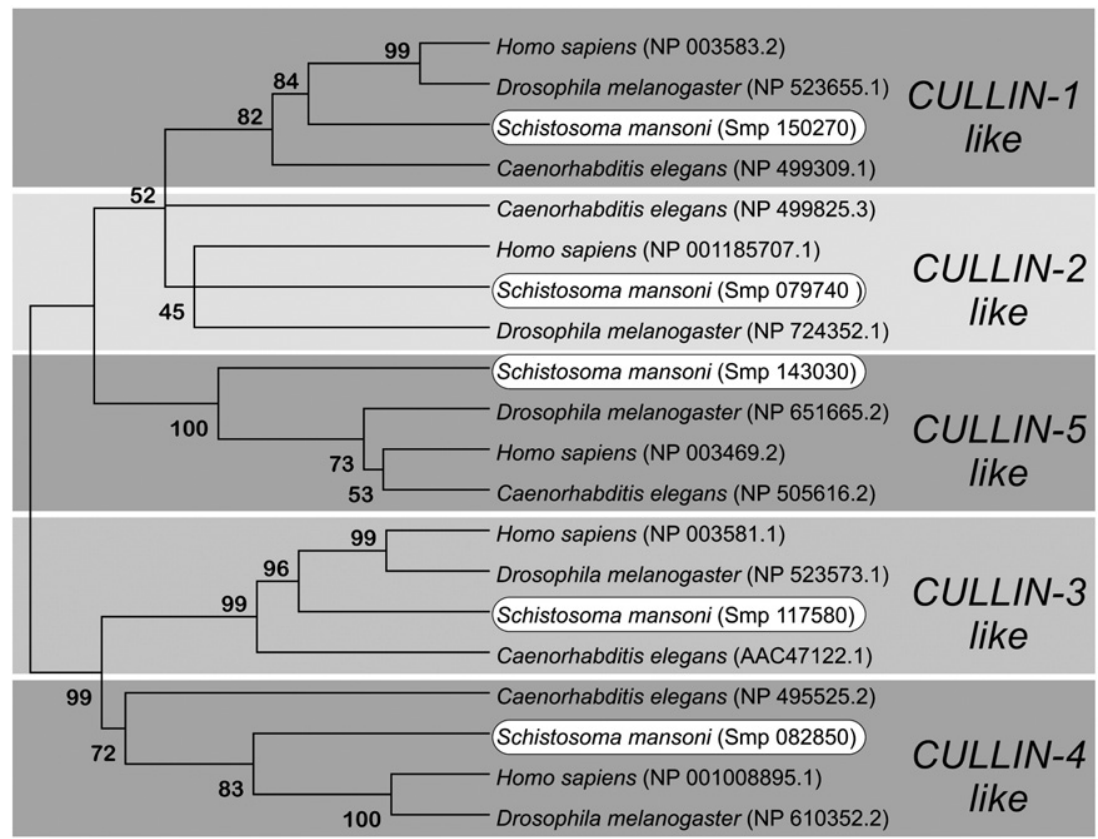

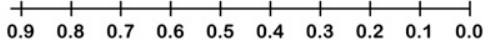

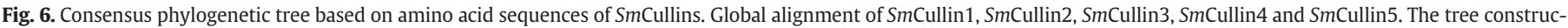

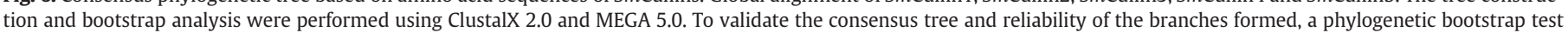
was used with 1000 replicates for each sequence, and reproduction of the branch in $50 \%$ of the runs considered the minimum for a reliable branch.

\section{Discussion}

Despite the advances in genome sequencing, transcriptome and proteome exploration, PTM in parasites is still poorly understood. This is a subject of fundamental importance because these pathways are involved in many cellular processes [18]. With regards to biochemical mechanisms, the NEDD8 pathway is similar to the PTM processes ubiquitylation and SUMOylation $[33,34]$.

Our in silico analysis revealed that the number of predicted NEDDylation pathway components in the S. mansoni genome is in agreement with other invertebrate genomes, suggesting an important functional conservation among these organisms. Regarding the $S$. japonicum genome, the number of enzymes involved in the NEDD8 conjugation process is the same as is found in S. mansoni, reinforcing the evolutive proximity between these species [35]. During this analysis the RBX protein was not found in the parasite EST database (http://www.genedb.org/genedb/smansoni/), despite the fact that this E3 ligase has been characterised in this helminth previously [36]. Furthermore, we have observed errors in the assembled genome sequence for a subunit of the E1 heterodimer (APP1), reinforcing the importance of re-annotating the putative proteins deposited in the genome database to increase data accuracy.

The NEDDylation pathway is conserved and essential for the viability of most model organisms, including Schizosaccharomyces pombe, C. elegans, Drosophila, Arabidopsis and mouse, supporting the hypothesis that NEDDylation is involved with important cellular processes in eukaryotic cells $[37,8,6,38,7,39]$. Based on these data, we decided to evaluate the conserved domains in predicted NEDD8 pathway components: NEDD8 (PF 11976), UBE2F (PF 00179) and SENP8 (PF 02902) (Fig. 1). Moreover, we analysed the conservation of E2 conjugation proteins by phylogenetic analysis and multiple sequence alignment. Two enzymes, UBC12 and UBE2F, have been previously identified as E2 conjugation proteins. By observing the phylogenetic tree, we have noted that the NEDD8-E2 from S. mansoni is unique and has higher identity with UBE2F than with UBE2M. In addition, they might share a common ancestral protein, suggesting that these proteins have been the result of gene duplication events. Biochemical and structural analyses have indicated how plasticity of hydrophobic E1-E2 interactions and E1 conformational flexibility allow one E1 to charge multiple E2s [40]. The E2 proteins have displayed distinct functions, with UBE2M/RBX1 and UBE2F/RBX2 each targeting different Cullins. UBE2M and UBE2F pairs specifically regulate NEDDylation of Cullins 1-4 and Cullin 5, respectively, in an E2-RING-dependent manner [40]. This specific mechanism in S. mansoni is still unclear because the parasite has a unique E2 and E3 ligase RBX. Furthermore, we found enhanced expression of Smube2f, a conjugation enzyme and partner of E1, at all examined developmental stages, and all components of the NEDDylation machinery were upregulated in the adult worm.

Recently it has been demonstrated that NEDDylation of Cullin targets is correlated with cell cycle progression. All the Cullin proteins are NEDDylated and involved in formation of various SCF complexes. The modification by NEDD8 induces a conformational change that inhibits the interaction with the inhibitory protein CAND1 and activates the ubiquitin E3 ligase [18]. Accordingly, we analysed the members of the Cullin family (Cullins $1-5$ ), the possible NEDDylation machinery targets. The conserved domains were characterised (Cullin: Pfam PF 00888 and Cullin-NEDD8: Pfam PF10557), confirming the presence of five Cullins in the S. mansoni genome, as well as their conservation. In addition, the expression profiles of these proteins during the transition from cercariae to adult worms were evaluated. Cullins showed similar expression profiles among analysed stages. Comparative analyses showed that Smcullin1 was the highest-expressed among the Cullins. Cullin1 is the most important among Cullins and has been shown to be involved in most E3ubiquitin ligase complexes [41], suggesting that most of the Cullin-RING ligases in S. mansoni have Cullin1 as their scaffold.

In addition to the Cullins, other NEDDylation machinery targets were analysed, such as the tumour suppressor proteins p63 and p73. Some reports showed NEDDylation inhibits transcriptional activities of these proteins, which are negative regulators of the cell cycle $[18,42]$. Recent studies showed that NEDDylation's role in p53 activity remains unclear. The NEDD8/ubiquitin interplay controls p53 subcellular 

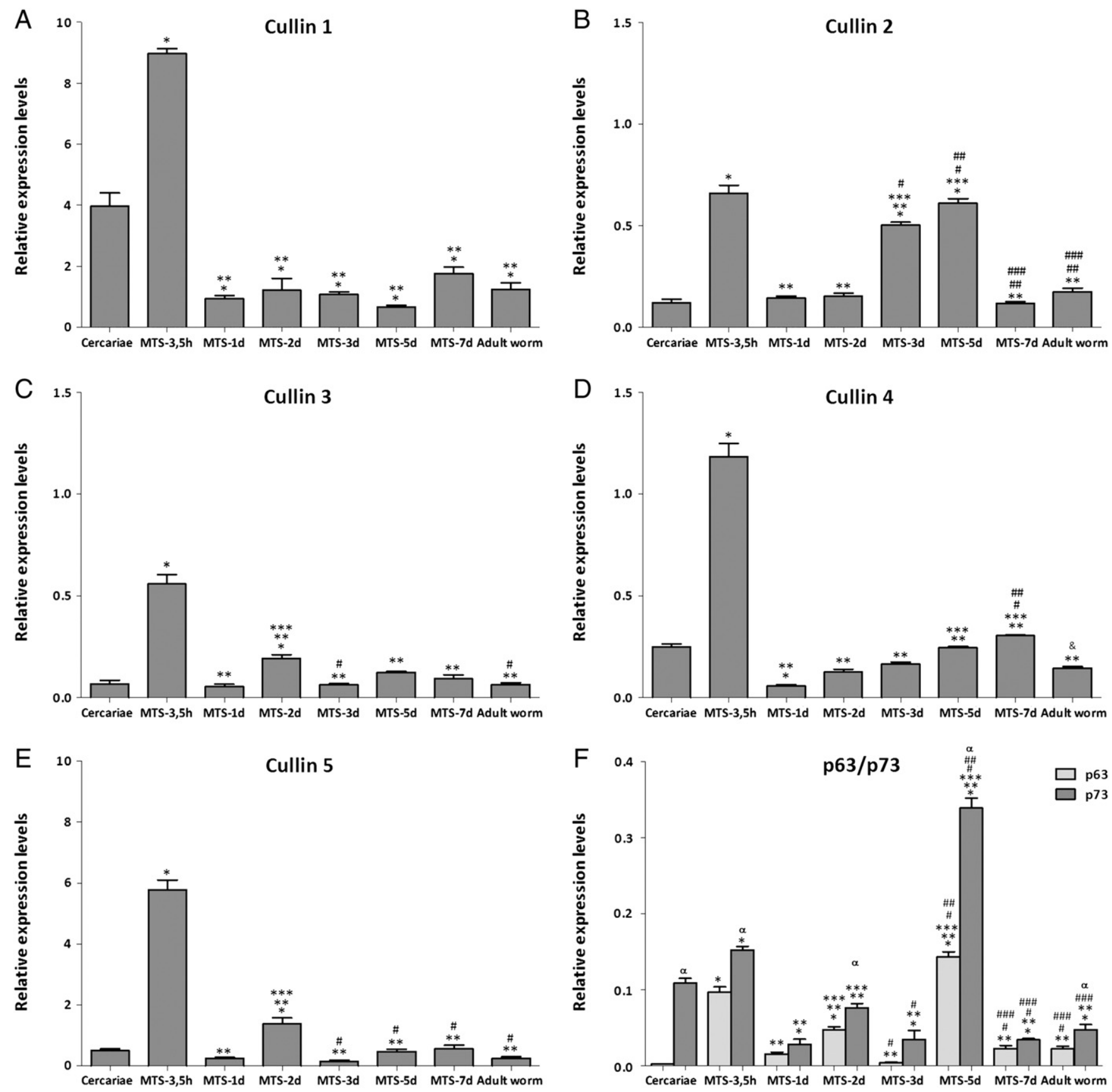

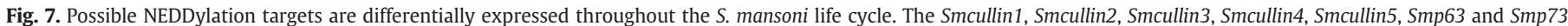

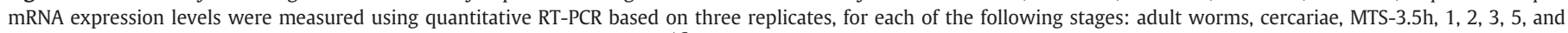

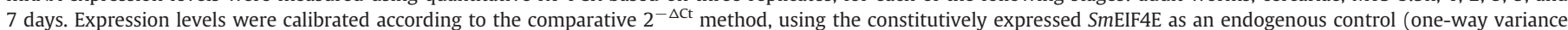

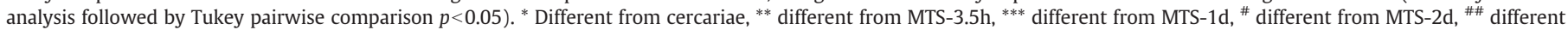
from MTS-3d, ${ }^{\# \# \# ~ d i f f e r e n t ~ f r o m ~ M T S-5 d ~ a n d ~}{ }^{\delta}$ different from MTS-7d. $t$ Tests were performed to compare p73 to p63 ( ${ }^{\alpha}$ different from p63).

localisation, suggesting that NEDD8 can control protein function $[43,44]$. By analysing the expression profiles of these transcripts, we observed that Smp73 is highly expressed compared to Smp63 in all parasite stages. Moreover, there was a 2-fold increase in p73 gene expression in cercariae levels compared to adult worms.

SENP8 has both hydrolase and isopeptidase activities, so this protease might process the NEDD8 precursor and promote deconjugation of NEDDylated substrates. However, under physiological conditions SENP8 is not able to DeNEDDylate Cullins, the major substrates of NEDDylation pathway. This process is catalysed by the CSN-5 subunit of the Cop9 signalosome complex (CSN) [18]. CSN is able to DeNEDDylate monoNEDDylated Cul1 under physiological conditions, but is not as efficient in deconjugating hyper- or polyNEDDylated Cul1. In contrast SENP8 is able to process hyper- but not monoNEDDylated Cul1 and is able to activate the NEDD8 pro-protein [16]. HyperNEDDylated Cul1 is induced in the cell under nonphysiological conditions, and thus may not represent a physiological target of either SENP8 or CSN-5. However, the ability of SENP8 to deconjugate hyperNEDDylated Cul1 likely derives from the ability of SENP8 to accommodate large leaving groups at the $C$ terminus of NEDD8. Although, nothing is known about the nature of the interactions between the Cullin component of these substrates and SENP8 or CSN-5 $[45,46]$. In the near future, this subunit of CSN will be investigated in this parasite. 
In conclusion, we described that NEDDylation machinery and presumable substrates were differentially expressed in S. mansoni. Considering that the transformation from cercariae to schistosomula requires the adaptation of the parasite to different environments and subsequent large scale cellular differentiation and growth, our data reinforce that the NEDD8 pathway is involved in parasite development. Additional studies for the mechanisms of NEDD8 conjugation in S. mansoni will provide further insights into the biological activities of this pathway and the role of NEDD8 conjugation as a rapid response to cercariae/schistosomula/adult worm development.

Supplementary data to this article can be found online at http:// dx.doi.org/10.1016/j.parint.2012.12.009.

\section{Acknowledgments}

The authors wish to thank the following transcriptome initiatives: São Paulo Transcriptome Consortium, Minas Gerais Genome Network, Wellcome Trust Genome Initiative (UK). This work was supported by the following Brazilian research agencies: FAPEMIG (Fundação de Amparo à Pesquisa do Estado de Minas Gerais, CBB 0558/09), NuBio UFOP (Núcleo de Bioinformática da Universidade Federal de Ouro Preto) and CNPq (Conselho Nacional de Desenvolvimento Científico e Tecnológico).

\section{References}

[1] Pickart CM, Eddins MJ. Ubiquitin: structures, functions, mechanisms. Biochimica et Biophysica Acta 2004;1695(1-3):55-72.

[2] Pickart CM, Fushman D. Polyubiquitin chains: polymeric protein signals. Current Opinion in Chemical Biology 2004;8(6):610-6

[3] Schwartz DC, Hochstrasser M. A superfamily of protein tags: ubiquitin, SUMO and related modifiers. Trends in Biochemical Sciences 2003;28(6):321-8.

[4] Kitahara R, Yamaguchi Y, Sakata E, Kasuya T, Tanaka K, Kato K, et al. Evolutionally conserved intermediates between ubiquitin and NEDD8. Journal of Molecular Biology 2006;363:395-404.

[5] Osaka F, Saeki M, Katayama S, Aida N, Toh-E A, Kominami K, et al. Covalent modifier NEDD8 is essential for SCF ubiquitin-ligase in fission yeast. EMBO Journal 2000; $13: 3475-84$.

[6] Tateishi K, Omata M, Tanaka K, Chiba T. The NEDD8 system is essential for cell cycle progression and morphogenetic pathway in mice. The Journal of Cell Biology 2001;155(4):571-9.

[7] Ou C, Lin Y, Chen Y, Chien C. Distinct protein degradation mechanisms mediated by Cul1 and Cul3 controlling Ci stability in Drosophila eye development. Genes \& Development 2002;18:2403-14.

[8] Osaka F, Kawasaki H, Aida N, Saeki M, Chiba T, Kawashima S, et al. A new NEDD8-ligating system for cullin-4A. Genes \& Development 1998;12:2263-8.

[9] Gong L, Yeh ET. Identification of the activating and conjugating enzymes of the NEDD8 conjugation pathway. Journal of Biological Chemistry 1999;274:12036-42.

[10] Kamura T, Conrad MN, Yan Q Conaway RC, Conaway JW. The Rbx1 subunit of SCF and VHL E3 ubiquitin ligase activates Rub1 modification of cullins Cdc53 and Cul2 Genes \& Development 1999;13:2928-33.

[11] Hori T, Osaka F, Chiba T, Miyamoto C, Okabayashi K, Shimbara N, et al. Covalent modification of all members of human cullin family proteins by NEDD8. Oncogene 1999;18:6829-34

[12] Ohki Y, Funatsu N, Konishi N, Chiba T. The mechanism of poly-NEDD8 chain formation in vitro. Biochemical and Biophysical Research Communications 2009;381:443-7.

[13] Jones J, Wu K, Yang Y, Guerrero C, Nillegoda N, Pan Z, et al. A targeted proteomic analysis of the ubiquitin-like modifier Nedd8 and associated proteins. Journal of Proteome Research 2008;7(3):1274-87.

[14] Lyapina S, Cope G, Shevchenko A, Serino G, Tsuge T, Zhou C, et al. Promotion of NEDD-CUL1 conjugate cleavage by COP9 signalosome. Science 2001;292:1382-5.

[15] Cope GA, Suh GS, Aravind L, Schwarz SE, Zipursky SL, Koonin EV, et al. Role of predicted metalloprotease motif of Jab1/Csn5 in cleavage of Nedd8 from Cul1. Science 2002;298:608-11.

[16] Mendoza HM, Shen L, Botting C, Lewis A, Chen J, Ink B, et al. NEDP1, a highly conserved cysteine protease that deNEDDylates Cullins. The Journal of Biological Chemistry 2003;278(28):25637-43.

[17] Pan ZQ, Kentsis A, Dias DC, Yamoah K, Wu K. Nedd8 on cullin: building an expressway to protein destruction. Oncogene 2004;23(11):1985-97.

[18] Rabut G, Peter M. Function and regulation of protein NEDDylation. 'Protein modifications: beyond the usual suspects' review series. EMBO Reports 2008;9:969-76.
[19] Guerra-Sa R, Castro-Borges W, Evangelista EA, Kettelhut IC, Rodrigues V. Schistosoma mansoni: functional proteasomes are required for development in the vertebrate host. Experimental Parasitology 2005;109:228-36.

[20] Mathieson W, Castro-Borges W, Wilson RA. The proteasome-ubiquitin pathway in the Schistosoma mansoni egg has development- and morphology-specific characteristics. Molecular and Biochemical Parasitology 2011;175:118-25.

[21] Castro-Borges W, Cartwright J, Ashton PD, Braschi S, Guerra Sa R, Rodrigues V, et al. The 20S proteasome of Schistosoma mansoni: a proteomic analysis. Proteomics 2007;7:1065-75.

[22] Cabral FJ, OSJr Pereira, Silva CS, Guerra-Sa R, Rodrigues V. Schistosoma mansoni encodes SMT3B and SMT3C molecules responsible for post-translational modification of cellular proteins. Parasitology International 2008;57:172-8.

[23] Pereira RV, Cabral FJ, Gomes MS, Baba EH, Jannotti-Passos LK, Carvalho O, et al. Molecular characterization of SUMO E2 conjugation enzyme: differential expression profile in Schistosoma mansoni. Parasitology Research 2011;109(6):1537-46.

[24] Harrop R, Wilson RA. Protein synthesis and release by cultured schistosomula of Schistosoma mansoni. Parasitology 1993;107:265-74.

[25] Basch P, DiConza J. In vitro development of Schistosoma mansoni cercariae. Journal of Parasitology 1977;63:245-9.

[26] Larkin MA, Blackshields G, Brown NP, Chenna R, McGettigan PA, McWilliam H, et al. Clustal W and Clustal X version 2.0. Bioinformatics 2007;23:2947-8.

[27] Kumar S, Tamura K, Nei M. MEGA3: integrated software for molecular evolutionary genetics analysis and sequence alignment. Briefings in Bioinformatics 2004;5: 150-63.

[28] Saitou N, Nei M. The neighbor-joining method: a new method for reconstructing phylogenetic trees. Molecular Biology and Evolution 1987;4:406-25.

[29] Liu S, Cai P, Hou N, Piao X, Wang H, Hung T, et al. Genome-wide identification and characterization of a panel of house-keeping genes in Schistosoma japonicum. Molecular and Biochemical Parasitology 2012;182(1-2):75-82

[30] Livak KJ, Schmittgen TD. Analysis of relative gene expression data using real-time quantitative PCR and the 2(-Delta Delta C(T)) Method. Methods 2001;25:402-8

[31] Tanaka H, Matsu-Ura T, Hirai H. The p53 expression and its developmental regulation in schistosomes. Memórias do Instituto Oswaldo Cruz 1992;87:71-3.

[32] Rutkowski R, Hofmann K, Gartner A. Phylogeny and function of the invertebrate p53 superfamily. Cold Spring Harbor Perspectives in Biology 2010;2(7):a001131.

[33] Kerscher O, Felberbaum R, Hochstrasser M. Modification of proteins by ubiquitin and ubiquitin-like proteins. Annual Review of Cell and Developmental Biology 2006;22:159-80.

[34] Kirkin V, Dikic I. Role of ubiquitin- and Ubl-binding proteins in cell signaling. Current Opinion in Cell Biology 2007;19:199-205.

[35] Young ND, Jex AR, Li B, Liu S, Yang L, Xiong Z, et al. Whole-genome sequence of Schistosoma haematobium. Nature Genetics 2012;44(2):221-8.

[36] Santos DN, Aguiar PHN, Lobo FP, Mourão MM, Tambor JH, Valadão AF, et al. Schistosoma mansoni: heterologous complementation of a yeast null mutant by SmRbx, a protein similar to a RING box protein involved in ubiquitylation. Experimental Parasitology 2007:116:440-9.

[37] Jones D, Candido EP. The NED-8 conjugating system in Caenorhabditis elegans is required for embryogenesis and terminal differentiation of the hypodermis. Developmental Biology 2000;226:152-65.

[38] Kurz T, Pintard L, Willis JH, Hamill DR, Gonczy P, Peter M, et al. Cytoskeletal regulation by the Nedd8 ubiquitin-like protein modification pathway. Science 2002;295:1294-8.

[39] Dharmasiri S, Dharmasiri N, Hellmann H, Estelle M. The RUB/Nedd8 conjugation pathway is required for early development in Arabidopsis. EMBO Journal 2003;22: 1762-70.

[40] Huang DT, Ayrault O, Hunt HW, Taherbhoy AM, Duda DM, Scott DC, et al. E2-RING expansion of the NEDD8 cascade confers specificity to cullin modification. Molecular Cell 2009;33(4):483-95.

[41] Merlet J, Burger J, Gomes JE, Pintard L. Regulation of cullin-RING E3 ubiquitinligases by NEDDylation and dimerization. Cellular and Molecular Life Sciences 2009;66(11-12):1924-38.

[42] Abida WM, Nikolaev A, Zhao W, Zhang W, Gu W. FBX011 promotes the NEDDylation of p53 and inhibits its transcriptional activity. Journal of Biological Chemistry 2007;282(3):1797-804.

[43] Carter S, Vousden KH. p53-Ubl fusions as models of ubiquitylation, sumoylation and NEDDylation of p53. Cell Cycle 2008;7(16):2519-28.

[44] Liu G, Xirodimas DP. NUB1 promotes cytoplasmic localization of p53 through cooperation of the NEDD8 and ubiquitin pathways. Oncogene 2010;29(15):2252-61.

[45] Gan-Erdene T, Nagamalleswari K, Yin L, Wu K, Pan ZQ, Wilkinson KD. Identification and characterization of DEN1, a deneddylase of the ULP family. Journal of Biological Chemistry 2003;278:28892-900.

[46] Wu K, Yamoah K, Dolios G, Gan-Erdene T, Tan P, Chen A, et al. DEN1 is a dual function protease capable of processing the $C$ terminus of Nedd 8 and deconjugating hyper-neddylated CUL1. Journal of Biological Chemistry 2003;278:28882-91. 